April, $1995 \quad$ MRR 036-95

hep-th/9506074

\title{
$O(n)$ model on the honeycomb lattice via reflection matrices: Surface critical behaviour
}

\author{
C. M. Yungf and M. T. Batchelor \\ Department of Mathematics, School of Mathematical Sciences \\ Australian National University, Canberra ACT 0200, Australia
}

\begin{abstract}
We study the $O(n)$ loop model on the honeycomb lattice with open boundary conditions. Reflection matrices for the underlying Izergin-Korepin $R$-matrix lead to three inequivalent sets of integrable boundary weights. One set, which has previously been considered, gives rise to the ordinary surface transition. The other two sets correspond respectively to the special surface transition and the mixed ordinary-special transition. We analyse the Bethe ansatz equations derived for these integrable cases and obtain the surface energies together with the central charges and scaling dimensions characterizing the corresponding phase transitions.
\end{abstract}

${ }^{*}$ Address after June 1, 1995: Research Institute of Mathematical Sciences, Kyoto University, Kyoto 606, Japan 


\section{Introduction}

The $O(n)$ model [1] in two dimensions has attracted considerable attention in recent years (see, e.g. Refs. [2-21] and references therein), not least because of its connection to the problem of planar self-avoiding walks or polymers in the $n \rightarrow 0$ limit [22]. Methods used to study the model have included, among others, Coulomb gas techniques [3, 4, 8], conformal invariance [5, 6, 17, 13, 15, 21], Bethe ansatz [9, 10, 11, 12, 19], bootstrap $S$ matrix 16, 17, 18, 20 and numerical transfer matrix calculations 114. The self-avoiding walk problem has also been extensively studied via a number of techniques (see, e.g. [23] and references therein). Of particular relevance here are the finite-size transfer matrix calculations [24, 25].

The critical behaviour of the $O(n)$ model is well understood, with an exact Bethe ansatz solution on the periodic honeycomb lattice [9, 10, 11 providing a confirmation of the earlier Coulomb gas and conformal invariance based results in the bulk. However, at least from the perspective of exactly solved lattice models, the situation is not as satisfactory for surface critical behaviour. A Bethe ansatz solution on the open honeycomb lattice was found [19], again confirming earlier results [5, 6, 23] for the ordinary surface transition [26]. However, predictions also exist [24, 15, 20, 21] for other surface transitions [26]. In this paper we derive exact results for the special surface transition, also on the honeycomb lattice, using a Bethe ansatz technique which utilises reflection matrices in an essential way [27]. We also derive exact results for the model with mixed ordinary and special boundary conditions. These results for the surface critical behaviour both complement and, in some cases, extend those currently available in the literature from other means. Our results for the special transition and its relevance to the problem of the adsorption transition for polymers were announced in [28].

The $O(n)$ or $n$-vector model on the honeycomb lattice is a statistical mechanical

model of $n$-dimensional unit vectors $\vec{S}_{i}$ living on the sites $i$ of the lattice and interacting via nearest neighbour couplings. Its partition function is given by

$$
Z^{\prime}=\int\left(\prod_{i} \mathrm{~d} \vec{S}_{i}\right) \exp \left(-\sum_{\langle i j\rangle} J_{i j} \vec{S}_{i} \cdot \vec{S}_{j}\right) .
$$

On an infinite lattice the coupling constant $J_{i j}$ is usually chosen to assume the same value $J$ for all nearest neighbour pairs $\langle i j\rangle$. In this paper we study the model with open boundary conditions (see Fig 1 ) with $J_{i j}$ taking values $J_{l}$ (respectively, $J_{r}$ ) if the nearest neighbour pair $\langle i j\rangle$ lives on the left (right) boundary. Following Domany et. al. [2], we argue that its critical behaviour is in common with that of a more amenable (but still $O(n)$-symmetric) model whose partition function is given by

$$
Z=\int\left(\prod_{i} \mathrm{~d} \vec{S}_{i}\right) \prod_{\langle i j\rangle}\left(1+\kappa_{i j} \vec{S}_{i} \cdot \vec{S}_{j}\right) .
$$




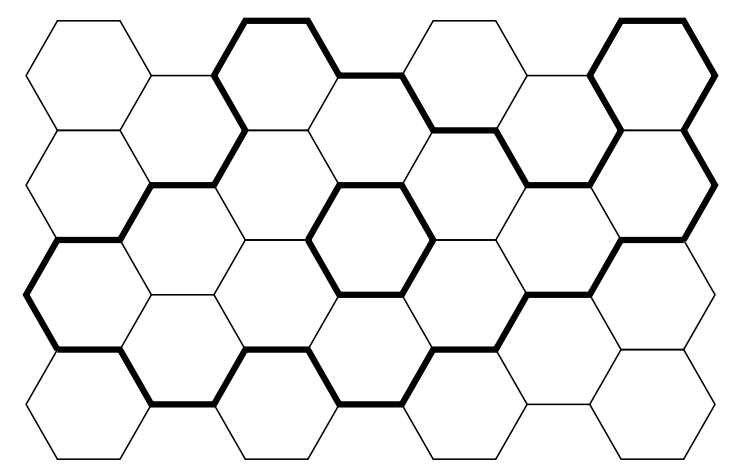

Figure 1: Honeycomb lattice of "width" $N=8$ on which the $O(n)$ model is defined. The lattice extends to infinity in both vertical directions. A typical configuration $\mathcal{G}$ contributing to the partition sum is shown in bold, with $P=2, N_{l}=2, N_{r}=4$ and $N_{b}=36$.

Here the nearest neighbour coupling constant $\kappa_{i j}$ takes values $\kappa_{l}$ (respectively, $\kappa_{r}$ ) if the pair $\langle i j\rangle$ lives on the left (right) boundary, and $\kappa$ otherwise.

Through a "high-temperature expansion" (in small $\kappa, \kappa_{l}$ and $\kappa_{r}$ ) $Z$ can be equivalently written as the partition sum

$$
Z=\sum_{\mathcal{G}} \kappa^{N_{b}} \kappa_{l}^{N_{l}} \kappa_{r}^{N_{r}} n^{P}
$$

over all configurations $\mathcal{G}$ of $P$ non-intersecting and closed loops of fugacity $n$ on the same lattice, with $N_{l}$ (respectively, $N_{r}$ ) bonds of $\mathcal{G}$ living on the left (right) boundary and $N_{b}$ bonds not living on either boundary. When $n \rightarrow 0$ only the empty graph contributes to the partition function. However, the $\ell$ lines joining the two points $\vec{x}$ and $\vec{y}$ in a "watermelon correlator" $G_{\ell}(\vec{x}-\vec{y})$ survives, giving rise in the continuum limit to the correlation function of $\ell$ self- and mutually avoiding walks tied at the ends.

The partition sum (1.3) can also be written in the following way which will eventually allow a mapping of the (non-local) loop model onto a (local) vertex model:

$$
Z \sim \sum_{\mathcal{G}} w_{1}^{N_{1}} \cdots w_{12}^{N_{12}} n^{P}
$$

where $w_{i}$ are Boltzmann weights for the allowed vertices depicted in Figure 2, of which there occurs $N_{i}$ such in the configuration $\mathcal{G}$. The weights $w_{i}$ are given by $w_{1}=\cdots=$ $w_{6}=1, w_{7}=w_{8}=\kappa^{-1} \equiv t, w_{9}=w_{10}=1, w_{11}=\kappa \kappa_{l}^{-2} \equiv t_{l}$ and $w_{12}=\kappa \kappa_{r}^{-2} \equiv t_{r}$. The partition sum (1.4) is defined up to an unimportant overall multiplicative factor.

For the $O(n)$ model on the (infinite) honeycomb lattice, the critical line

$$
\kappa^{-1}=\left(2+(2-n)^{1 / 2}\right)^{1 / 2}
$$




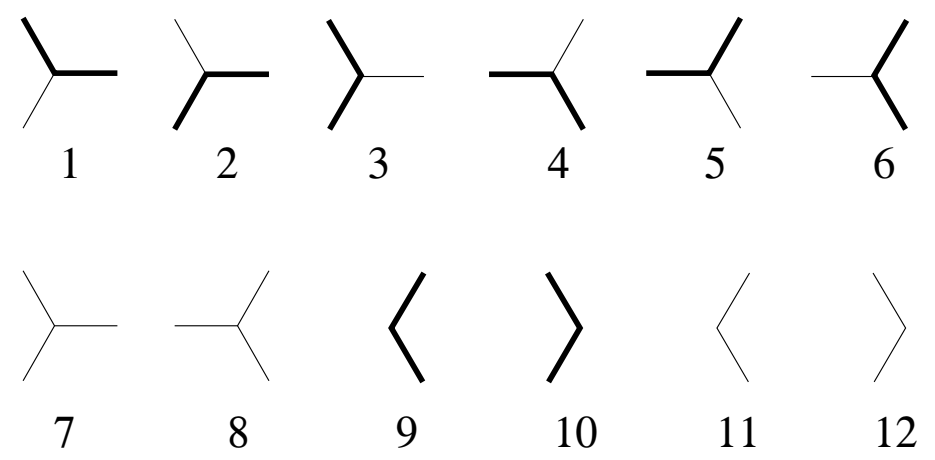

Figure 2: Allowed loop vertices for the honeycomb loop model. Vertex $i$ carries a Boltzmann weight $w_{i}$.

was first obtained by Nienhuis [3] using a mapping to various models and the Coulomb gas. Baxter [9] subsequently showed that a vertex model to which the loop model (1.3) can be mapped is solvable by the coordinate Bethe ansatz precisely along the line (1.5). The Bethe ansatz solution was then used to derive critical properties for the $O(n)$ model in Refs. 10, 11]. For instance, in the parametrization

$$
\begin{aligned}
n & =-2 \cos \pi g \\
\kappa^{-1}=t & =2 \sin \frac{\pi g}{4},
\end{aligned}
$$

the model has central charge

$$
c=1-\frac{6(1-g)^{2}}{g} .
$$

For the open honeycomb lattice of relevance to this paper, Batchelor and Suzuki [19] showed that along the line specified by (1.5) and, in addition, $\kappa_{l}=\kappa_{r}=\kappa$, the model is likewise solvable by the coordinate Bethe ansatz and corresponds to the $O(n)$ model at the ordinary surface transition. The Bethe ansatz analysis showed that the central charge (1.8) remains valid. Furthermore, the model has the set of scaling dimensions

$$
X_{\ell}^{\mathrm{O}-\mathrm{O}}=h_{\ell+1,1}
$$

in agreement with an earlier identification in terms of the Kac formula, where [6]

$$
h_{p, q}=\frac{1}{4} g p^{2}-\frac{1}{2} p q+\frac{q^{2}-(g-1)^{2}}{4 g} .
$$

Physically, this set of geometric dimensions characterizes the algebraic decay of the half-watermelon correlators $G_{\ell}(\vec{x}-\vec{y})$ at criticality [6]. 
The present authors have recently presented a systematic approach [27] to obtain integrable loop models with open boundaries. This approach begins with Sklyanin's extension of the Quantum Inverse Scattering Method to obtain integrable open spin chains [29, 30]. By incorporating inhomogeneities and making them alternate with the spectral parameter [31] the Sklyanin transfer matrix becomes relevant to vertex models with open boundaries. For certain choices of $K$-matrices, or solutions to the reflection equation, as input to the Sklyanin transfer matrix the corresponding vertex model can be mapped to a loop model.

The $O(n)$ model of interest here can be obtained from a special case of the IzerginKorepin [32] or $A_{2}^{(2)}$ vertex model [33, 34] (see also the reference to Korepin in Ref. [9]). There are three inequivalent diagonal $K$-matrices associated to the $A_{2}^{(2)} R$-matrix [35]. Of these the two giving rise to non-quantum group invariant spin chains allow a mapping to loop models. We showed in [27] how to recover the model of [19] which corresponds to the ordinary surface transition. In addition we also found a different set of boundary weights for which the model is integrable; these correspond to

$$
\kappa_{l}=\kappa_{r}=\left(-2 \cos \frac{\pi g}{2}\right)^{-1 / 2} \equiv \kappa_{\mathrm{Sp}} .
$$

Note that this critical coupling is related to $n$ through

$$
\kappa_{S p}=(2-n)^{-1 / 4} .
$$

We shall presently argue that this solution corresponds to the $O(n)$ model at the special transition, with central charge ([1.8) and the set of scaling dimensions

$$
X_{\ell}^{\mathrm{Sp}-\mathrm{Sp}}=h_{\ell+1,3},
$$

confirming a conjecture using bootstrap boundary $S$-matrices [20]. We also obtain a third set of integrable boundary weights which is a mixture of the first two; namely,

$$
\kappa_{l}=\kappa, \quad \kappa_{r}=\kappa_{\mathrm{Sp}}
$$

We argue that this corresponds to the $O(n)$ model with mixed ordinary-special boundary conditions, with central charge

$$
c^{\mathrm{O}-\mathrm{Sp}}=1-\frac{6(2-g)^{2}}{g},
$$

or equivalently $c^{\mathrm{O}-\mathrm{Sp}}=c-24 t^{\mathrm{O}-\mathrm{Sp}}$ with the "mixed-boundary" scaling index given by $t^{\mathrm{O}-\mathrm{Sp}}=h_{1,2}$, confirming a recent result obtained using conformal invariance [21]. In addition, we find the set of scaling dimensions

$$
X_{\ell}^{\mathrm{O}-\mathrm{Sp}}=h_{\ell+1,1}-\frac{1}{2} \ell .
$$

A brief summary of our results for mixed boundaries and their relevance to twodimensional polymers has been given elsewhere [36]. 


\section{Honeycomb limit of the $A_{2}^{(2)}$ loop model}

In this section we present the derivation of integrable $O(n)$ loop models on the honeycomb lattice with open boundaries from the $A_{2}^{(2)} R$-matrix and associated diagonal $K$-matrices [35]. This involves the following steps: (I) Interpreting the Sklyanin transfer matrix $t(u, \boldsymbol{\omega})$ [29] with alternating inhomogeneities [31] $\boldsymbol{\omega}$ as a diagonal-to-diagonal transfer matrix $t_{\mathrm{D}}(u)$ for a vertex model on the square lattice with open boundaries. (II) Mapping the vertex model to a loop model on the same lattice. (III) Taking the 'the honeycomb limit' to arrive at the honeycomb lattice loop model and its transfer matrix $t_{\mathrm{H}}$. Via the same chain of mappings the eigenvalue expression for $t(u, \boldsymbol{\omega}$ ) (and associated Bethe ansatz equations) gives rise to the corresponding expression for $t_{\mathrm{H}}$. This procedure has been explained in detail in our previous paper [27 for several threestate $R$-matrices. In particular, the $O(n)$ models with non-mixed boundaries to which we hitherto refer as $\mathrm{O}-\mathrm{O}$ and $\mathrm{Sp}-\mathrm{Sp}$ are treated therein. We therefore concentrate here on the mixed boundary case denoted by O-Sp.

\subsection{Sklyanin transfer matrix and eigenvalue expression}

The $R$-matrix for the $A_{2}^{(2)}$ model 32] is given in terms of a spectral parameter $u$ and anisotropy $\lambda$ by

$$
R(u)=\left(\begin{array}{lll|ll|lll}
c & & & & & & \\
& b & & e & & & & \\
& & d & & g & & f & \\
\hline \bar{e} & & b & & & & \\
& & \bar{g} & & a & & g & \\
& & & b & & e & \\
\hline & \bar{f} & & \bar{g} & & d & & \\
& & & & \bar{e} & & b & \\
& & & & & & c
\end{array}\right),
$$

where

$$
\begin{aligned}
a(u) & =\sinh (u-3 \lambda)-\sinh (5 \lambda)+\sinh (3 \lambda)+\sinh (\lambda), \\
b(u) & =\sinh (u-3 \lambda)+\sinh (3 \lambda), \\
c(u) & =\sinh (u-5 \lambda)+\sinh (\lambda), \\
d(u) & =\sinh (u-\lambda)+\sinh (\lambda), \\
e(u) & =-2 e^{-\frac{u}{2}} \sinh (2 \lambda) \cosh \left(\frac{u}{2}-3 \lambda\right), \\
\bar{e}(u) & =-2 e^{\frac{u}{2}} \sinh (2 \lambda) \cosh \left(\frac{u}{2}-3 \lambda\right), \\
f(u) & =-2 e^{-u+2 \lambda} \sinh (\lambda) \sinh (2 \lambda)-e^{-\lambda} \sinh (4 \lambda),
\end{aligned}
$$




$$
\begin{aligned}
\bar{f}(u) & =2 e^{u-2 \lambda} \sinh (\lambda) \sinh (2 \lambda)-e^{\lambda} \sinh (4 \lambda), \\
g(u) & =2 e^{-\frac{u}{2}+2 \lambda} \sinh \left(\frac{u}{2}\right) \sinh (2 \lambda) \\
\bar{g}(u) & =-2 e^{\frac{u}{2}-2 \lambda} \sinh \left(\frac{u}{2}\right) \sinh (2 \lambda) .
\end{aligned}
$$

It was shown in [35] that there exists three inequivalent diagonal $K$-matrices $K^{-}(u)$ satisfying the reflection equation

$$
\begin{aligned}
& R_{12}(u-v) \stackrel{1}{K^{-}}(u) R_{21}(u+v) \stackrel{2}{K^{-}}(v)= \\
& 2 \\
& K^{-}(v) R_{12}(u+v) \stackrel{1}{K^{-}}(u) R_{21}(u-v) .
\end{aligned}
$$

They are namely, $K^{-}(u)=1$ and $K^{-}(u)=\Gamma^{ \pm}(u)$, where

$$
\Gamma^{ \pm}(u)=\operatorname{diag}\left(e^{-u} \psi^{ \pm}(u), \phi^{ \pm}(u), e^{u} \psi^{ \pm}(u)\right)
$$

with

$$
\begin{aligned}
& \psi^{ \pm}(u)=\cosh \left(\frac{u}{2}-3 \lambda\right) \pm \mathrm{i} \sinh \left(\frac{u}{2}\right) \\
& \phi^{ \pm}(u)=\cosh \left(\frac{u}{2}+3 \lambda\right) \mp \mathrm{i} \sinh \left(\frac{u}{2}\right) .
\end{aligned}
$$

Let $K^{+}(u)=K^{-}(-u-\eta)^{t} M$, where $K^{-}(u)$ is any of the three $K$-matrices defined above, $\eta=-6 \lambda-\mathrm{i} \pi$ is the crossing parameter and $M=\operatorname{diag}\left(e^{2 \lambda}, 1, e^{-2 \lambda}\right)$ is the crossing matrix. Then the Sklyanin transfer matrix [29, 30]

$$
t(u, \boldsymbol{\omega})=\operatorname{tr}_{a} \stackrel{a}{K^{+}}(u) T_{a}(u, \boldsymbol{\omega}) \stackrel{a}{K^{-}}(u) \tilde{T}_{a}(u, \boldsymbol{\omega}),
$$

forms a commuting family: $[t(u, \boldsymbol{\omega}), t(v, \boldsymbol{\omega})]=0$ for arbitrary $u, v$. Here $T_{a}$ and $\tilde{T}_{a}$ are monodromy matrices defined by

$$
\begin{aligned}
T_{a}(u, \boldsymbol{\omega}) & =R_{a 1}\left(u+\omega_{1}\right) R_{a 2}\left(u+\omega_{2}\right) \cdots R_{a N}\left(u+\omega_{N}\right), \\
\tilde{T}_{a}(u, \boldsymbol{\omega}) & =R_{N a}\left(u-\omega_{N}\right) \cdots R_{2 a}\left(u-\omega_{2}\right) R_{1 a}\left(u-\omega_{1}\right),
\end{aligned}
$$

where $a$ labels the auxiliary space $V_{a}$ and $1, \ldots, N$ label quantum spaces. With three choices each for $K^{-}(u)$ and $K^{+}(u)$ we have therefore nine possiblities for $t(u, \boldsymbol{\omega})$. The three non-mixed cases were dealt with in [27]. It was shown there that the non quantum group-invariant cases, $\mathrm{O}-\mathrm{O}$ and $\mathrm{Sp}-\mathrm{Sp}$, based on $\Gamma^{ \pm}(u)$ give rise to loop models, in contrast to the quantum group-invariant case with $K^{-}(u)=1, K^{+}(u)=M$. Similar reasoning shows that only two of the mixed cases O-Sp and Sp-O give rise to loop models. These two loop models are equivalent, as to be expected, and we henceforth deal only with the case O-Sp specified by $K^{-}(u)=\Gamma^{-}(u)$ and $K^{+}(u)=\Gamma^{+}(-u-\eta)^{t} M$. 
The eigenvalue expression and Bethe ansatz equations for the Sklyanin transfer matrix $t(u, \boldsymbol{\omega})$ were obtained for the cases O-O and Sp-Sp in [27] using what can be referred to as the "Doubling Hypothesis" (cf. [37, 38]). For the model at hand, this says that the eigenvalue expression can be written in the form

$$
\Lambda(u, \boldsymbol{\omega})=\sum_{i=1}^{3} \alpha_{i}(u) \Phi_{i}(u, \boldsymbol{\omega}) A_{i}(u),
$$

where only the "boundary contributions" $\alpha_{i}(u)$ vary with the chosen (diagonal) $K$ matrices. The functions $\Phi_{i}(u, \boldsymbol{\omega})$ and $A_{i}(u)$ are doubled versions of the corresponding functions which appear in the eigenvalue expression for the transfer matrix with periodic boundary conditions. For the $A_{2}^{(2)}$ model they are given by

$$
\begin{aligned}
\Phi_{1}(u, \boldsymbol{\omega}) & =\prod_{i=1}^{N} c\left(u+\omega_{i}\right) c\left(u-\omega_{i}\right), \\
\Phi_{2}(u, \boldsymbol{\omega}) & =\prod_{i=1}^{N} b\left(u+\omega_{i}\right) b\left(u-\omega_{i}\right), \\
\Phi_{3}(u, \boldsymbol{\omega}) & =\prod_{i=1}^{N} d\left(u+\omega_{i}\right) d\left(u-\omega_{i}\right),
\end{aligned}
$$

where $b(u), c(u), d(u)$ are diagonal matrix elements of $R(u)$ and

$$
\begin{aligned}
A_{1}(u)= & \prod_{j=1}^{M} \frac{\sinh \left[\frac{1}{2}\left(u+u_{j}\right)+\lambda\right] \sinh \left[\frac{1}{2}\left(u-u_{j}\right)+\lambda\right]}{\sinh \left[\frac{1}{2}\left(u+u_{j}\right)-\lambda\right] \sinh \left[\frac{1}{2}\left(u-u_{j}\right)-\lambda\right]} \\
A_{2}(u)= & \prod_{j=1}^{M} \frac{\sinh \left[\frac{1}{2}\left(u+u_{j}\right)-3 \lambda\right] \sinh \left[\frac{1}{2}\left(u-u_{j}\right)-3 \lambda\right]}{\sinh \left[\frac{1}{2}\left(u+u_{j}\right)-\lambda\right] \sinh \left[\frac{1}{2}\left(u-u_{j}\right)-\lambda\right]} \\
& \times \prod_{j=1}^{M} \frac{\cosh \left[\frac{1}{2}\left(u+u_{j}\right)\right] \cosh \left[\frac{1}{2}\left(u-u_{j}\right)\right]}{\cosh \left[\frac{1}{2}\left(u+u_{j}\right)-2 \lambda\right] \cosh \left[\frac{1}{2}\left(u-u_{j}\right)-2 \lambda\right]}, \\
A_{3}(u)= & \prod_{j=1}^{M} \frac{\cosh \left[\frac{1}{2}\left(u+u_{j}\right)-4 \lambda\right] \cosh \left[\frac{1}{2}\left(u-u_{j}\right)-4 \lambda\right]}{\cosh \left[\frac{1}{2}\left(u+u_{j}\right)-2 \lambda\right] \cosh \left[\frac{1}{2}\left(u-u_{j}\right)-2 \lambda\right]} .
\end{aligned}
$$

The boundary contributions $\alpha_{i}(u)$ are determined by the action of $t(u, \boldsymbol{\omega})$ on the reference state $|\Omega\rangle$, being the $N$-fold tensor product of the vector with 1 as its first entry and 0 elsewhere, and depend on the $K$-matrices $K^{ \pm}(u)$ according to [27]

$$
\begin{aligned}
& \alpha_{1}(u)=K_{11}^{-}\left\{K_{11}^{+}+\frac{K_{22}^{+} \bar{e}^{2}}{c^{2}-b^{2}}+\frac{K_{33}^{+}}{c^{2}-d^{2}}\left(\bar{f}^{2}+\frac{\bar{g}^{2} \bar{e}^{2}}{c^{2}-b^{2}}\right)\right\}, \\
& \alpha_{2}(u)=K_{22}^{+} K_{22}^{-}-\frac{K_{22}^{+} K_{11}^{-} \bar{e}^{2}}{c^{2}-b^{2}}-\frac{K_{33}^{+} K_{22}^{-} \bar{g}^{2}}{d^{2}-b^{2}}-\frac{K_{33}^{+} K_{11}^{-} \bar{g}^{2} \bar{e}^{2}}{\left(c^{2}-b^{2}\right)\left(b^{2}-d^{2}\right)}, \\
& \alpha_{3}(u)=K_{33}^{+}\left\{K_{33}^{-}+\frac{K_{22}^{-} \bar{g}^{2}}{d^{2}-b^{2}}-\frac{K_{11}^{-}}{c^{2}-d^{2}}\left(\bar{f}^{2}-\frac{\bar{g}^{2} \bar{e}^{2}}{b^{2}-d^{2}}\right)\right\} .
\end{aligned}
$$


Here $\bar{e}, c$, etc. are matrix elements of $R(u)$ whose dependence on $u$, like those of the matrix elements of $K^{ \pm}(u)$, have been suppressed for convenience. For O-Sp boundaries we find the following:

$$
\begin{aligned}
\alpha_{1}(u) & =\frac{\sinh (u-6 \lambda)}{c(2 u)} \psi^{+}(u) \psi^{-}(u) \xi^{+}(u), \\
\alpha_{2}(u) & =\frac{\sinh (u-6 \lambda) \sinh (u)}{c(2 u) \sinh (u-4 \lambda)} \psi^{+}(u) \psi^{-}(u) \xi^{+}(u), \\
\alpha_{3}(u) & =\frac{\sinh (u) \sinh (u-2 \lambda)}{c(2 u) \sinh (u-4 \lambda)} \psi^{+}(u) \psi^{-}(u) \xi^{+}(u),
\end{aligned}
$$

where

$$
\xi^{ \pm}(u)=2(\cosh (3 \lambda-u) \pm \mathrm{i} \sinh 2 \lambda)
$$

and $\psi^{ \pm}(u)$ is as defined in Eq. (2.4). Together with Eqs. (2.10) and (2.11) these fully determine the eigenvalue $\Lambda(u, \boldsymbol{\omega})$ through Eq. (2.9). The corresponding Bethe ansatz equations are obtained by requiring $\Lambda(u, \boldsymbol{\omega})$ to be analytic in $u$. It should be pointed out that our justification for the eigenvalue expression (2.9) is not as strong as we would like, although we have no doubt about its correctness; a derivation using the algebraic Bethe ansatz [29] would be preferable. In fact, a derivation using the analytic Bethe ansatz generalizaling the study of [39] for the quantum group-invariant case would also go a long way towards answering this criticism. At present our result is backed up mainly with numerical checks (see end of Section 2).

\section{$2.2 \quad$ Vertex and loop models with open boundaries}

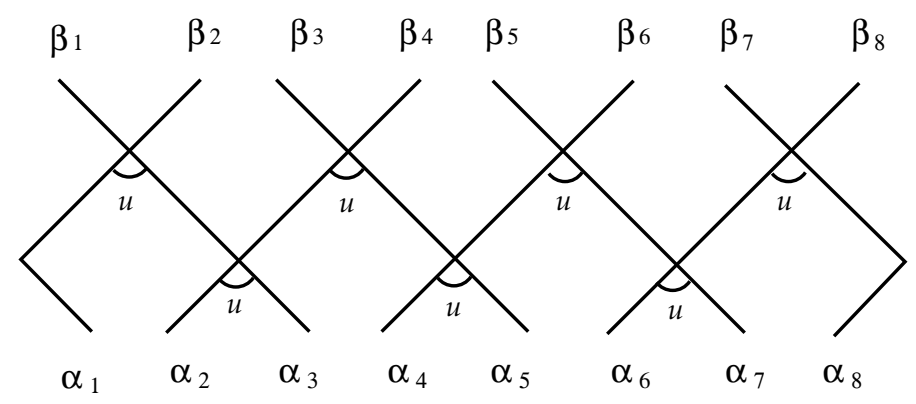

Figure 3: The "diagonal-to-diagonal" transfer matrix $t_{D}(u)$ which takes the state $\left(\alpha_{1}, \ldots, \alpha_{N}\right)$ to the state $\left(\beta_{1}, \ldots, \beta_{N}\right)$. All internal edges are summed over.

When the inhomogeneities $\boldsymbol{\omega}$ are chosen to alternate as $\omega_{i}=(-)^{i+1} u$, the Sklyanin transfer matrix turns into the diagonal-to-diagonal transfer matrix $t_{\mathrm{D}}(u)$ for a vertex 
model on the square lattice with open boundaries [27] (see also [31]). More specifically, define the functions

$$
\begin{aligned}
l_{a}^{b}(u) & =\sum_{c d} R_{d a}^{b c}(2 u) K_{c d}^{+}(u), \\
r_{a}^{b}(u) & =K_{a b}^{-}(u) .
\end{aligned}
$$

Then the object

$$
t_{\mathrm{D}}(u)=\frac{t\left(\frac{u}{2}, \omega_{j}=(-)^{j+1} \frac{u}{2}\right) R_{11}^{11}(u)}{\Phi_{1}\left(\frac{u}{2}, \omega_{j}=(-)^{j+1} \frac{u}{2}\right) l_{1}^{1}\left(\frac{u}{2}\right) r_{1}^{1}\left(\frac{u}{2}\right)},
$$

is the diagonal-to-diagonal transfer matrix (see Fig. 3) for the vertex model with bulk weights given by

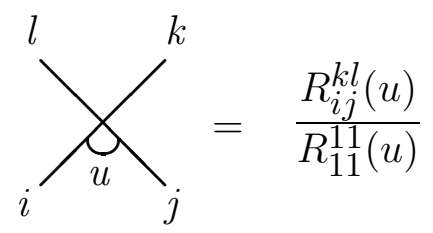

and boundary weights given by

$$
\left.\searrow_{i}^{j}=\frac{l_{i}^{j}(u / 2)}{l_{1}^{1}(u / 2)}, \quad{ }_{i}^{j}\right\rangle=\frac{r_{i}^{j}(u / 2)}{r_{1}^{1}(u / 2)}
$$

For alternating inhomogeneities $\omega_{i}=(-)^{i+1} u$ we note that $\Phi_{2}(u, \boldsymbol{\omega})$ and $\Phi_{3}(u, \boldsymbol{\omega})$ vanish due to $b(0)=d(0)=0$. Using the identity $\alpha_{1}(u)=l_{1}^{1}(u) r_{1}^{1}(u) / R_{11}^{11}(u)$ we arrive at the eigenvalue expression

$$
t_{\mathrm{D}}(u)=A_{1}\left(\frac{u}{2}\right)
$$

with the function $A_{1}(u)$ defined in (2.11). The associated Bethe ansatz equations are those which render $\Lambda(u, \boldsymbol{\omega})$ analytic in $u$, but with the inhomogeneities subsequently specialized to $\omega_{j}=(-)^{j+1} u$. Note that the order is crucial; analyticity of $t_{\mathrm{D}}(u)$ does not give the Bethe ansatz equations. With this we accomplish step (I).

We shall presently make a change of variables $u \rightarrow 2 \mathrm{i} u, \lambda \rightarrow \mathrm{i} \lambda+\mathrm{i} \pi / 2$, which takes us into the critical regime of the model for real $u, \lambda$. The eigenvalue expression for $t_{\mathrm{D}}(u)$ becomes (after a shift $u_{j} \rightarrow u_{j}+\mathrm{i} \pi$ )

$$
\Lambda_{\mathrm{D}}(u)=\prod_{j=1}^{M} \frac{\sinh \left[\frac{1}{2}\left(\mathrm{i} u+u_{j}\right)+\mathrm{i} \lambda\right] \sinh \left[\frac{1}{2}\left(\mathrm{i} u-u_{j}\right)+\mathrm{i} \lambda\right]}{\sinh \left[\frac{1}{2}\left(\mathrm{i} u+u_{j}\right)-\mathrm{i} \lambda\right] \sinh \left[\frac{1}{2}\left(\mathrm{i} u-u_{j}\right)-\mathrm{i} \lambda\right]}
$$


while the Bethe ansatz equations now take the form $(k=1, \ldots, M)$

$$
\begin{aligned}
\frac{\sinh \left(\mathrm{i} \lambda-u_{k}\right)}{\sinh \left(\mathrm{i} \lambda+u_{k}\right)}\left(\frac{\sinh \left[\frac{1}{2}\left(u_{k}-2 \mathrm{i} \lambda-\mathrm{i} u\right)\right] \sinh \left[\frac{1}{2}\left(u_{k}-2 \mathrm{i} \lambda+\mathrm{i} u\right)\right]}{\sinh \left[\frac{1}{2}\left(u_{k}+2 \mathrm{i} \lambda+\mathrm{i} u\right)\right] \sinh \left[\frac{1}{2}\left(u_{k}+2 \mathrm{i} \lambda-\mathrm{i} u\right)\right]}\right)^{N} \\
=\prod_{j \neq k}^{M} \frac{\sinh \left[\frac{1}{2}\left(u_{k}+u_{j}\right)-2 \mathrm{i} \lambda\right] \sinh \left[\frac{1}{2}\left(u_{k}-u_{j}\right)-2 \mathrm{i} \lambda\right]}{\sinh \left[\frac{1}{2}\left(u_{k}+u_{j}\right)+2 \mathrm{i} \lambda\right] \sinh \left[\frac{1}{2}\left(u_{k}-u_{j}\right)+2 \mathrm{i} \lambda\right]} \\
\\
\times \frac{\sinh \left[\frac{1}{2}\left(u_{k}+u_{j}\right)+\mathrm{i} \lambda\right] \sinh \left[\frac{1}{2}\left(u_{k}-u_{j}\right)+\mathrm{i} \lambda\right]}{\sinh \left[\frac{1}{2}\left(u_{k}+u_{j}\right)-\mathrm{i} \lambda\right] \sinh \left[\frac{1}{2}\left(u_{k}-u_{j}\right)-\mathrm{i} \lambda\right]}
\end{aligned}
$$

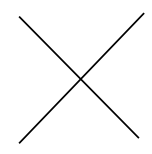

1

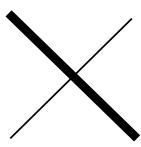

7

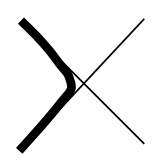

2

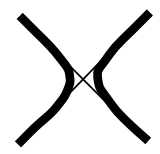

8

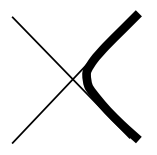

3

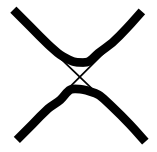

9

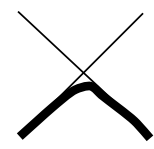

4

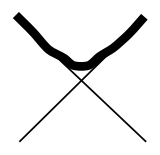

5

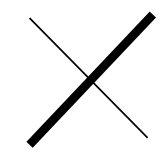

6

Figure 4: Allowed vertices for the loop model with partition function $Z_{\text {loop }}$. Vertex $i$ carries Boltzmann weight $\rho_{i}$.

Using an argument very similar to that for O-O and Sp-Sp [27] one can show that the vertex model partition function is equivalent to that of a loop model on the same lattice

$$
Z_{\text {loop }}=\sum_{\mathcal{G}} \rho_{1}^{N_{1}} \cdots \rho_{13}^{N_{13}} n^{P}
$$

where the sum is over all configurations $\mathcal{G}$ of non-intersecting closed loops which cover some (or none) of the edges, with non-zero Boltzmann weights $\rho_{i}$ shown in Figure 4. In the configuration $\mathcal{G}, N_{i}$ is the number of occurences of the weight of type $i$ while $P$ is the total number of closed loops of fugacity $n$. The explicit expressions for $\rho_{i}$ and $n$ are given (in the shifted variables $u, \lambda$ ) by

$$
\begin{aligned}
\rho_{1} & =[\sin (3 \lambda-u) \sin (u)+\sin (2 \lambda) \sin (3 \lambda)][\sin (3 \lambda-u) \sin (2 \lambda-u)]^{-1}, \\
\rho_{2}=\rho_{3} & =\epsilon_{1} \sin (2 \lambda) \sin (2 \lambda-u)^{-1}, \\
\rho_{4}=\rho_{5} & =\epsilon_{2} \sin (u) \sin (2 \lambda)[\sin (3 \lambda-u) \sin (2 \lambda-u)]^{-1}, \\
\rho_{6}=\rho_{7} & =\sin (u) \sin (2 \lambda-u)^{-1},
\end{aligned}
$$




$$
\begin{aligned}
\rho_{8} & =1 \\
\rho_{9} & =-\sin (\lambda-u) \sin (u)[\sin (3 \lambda-u) \sin (2 \lambda-u)]^{-1} \\
\rho_{10} & =\epsilon_{1} e^{-\mathrm{i} u} \\
\rho_{11} & =e^{-\mathrm{i} u} \sin \left[\frac{1}{2}(3 \lambda+u)\right] \sin \left[\frac{1}{2}(3 \lambda-u)\right]^{-1} \\
\rho_{12} & =\epsilon_{1} e^{\mathrm{i} u} \\
\rho_{13} & =e^{\mathrm{i} u} \cos \left[\frac{1}{2}(3 \lambda+u)\right] \cos \left[\frac{1}{2}(3 \lambda-u)\right]^{-1} \\
n & =-2 \cos (4 \lambda)
\end{aligned}
$$

where $\epsilon_{1}^{2}=\epsilon_{2}^{2}=1$. This concludes step (II).

To accomplish step (III) we now choose $\epsilon_{1}=\epsilon_{2}=1$ and take the "honeycomb limit" $u=\lambda$. As seen from Eqs. (2.23), the Boltzmann weight $\rho_{9}$ vanishes in this limit allowing the remaining bulk vertices to be "pulled apart horizontally" to give the vertices of Figure 5. It is apparent that these latter vertices define a honeycomb lattice loop model. In other words, in the honeycomb limit the square lattice loop model defined through the weights of Figure 4 is equivalent to the honeycomb lattice loop model defined through the weights of Figure 5 . There is in fact a second honeycomb limit, which we will consider no further, where $\rho_{8}$ vanishes and the remaining bulk vertices are pulled apart vertically.

The left and right boundary weights can be normalized separately such that $\rho_{10}=$ $\rho_{12}=1$, while keeping $Z_{\text {loop }}$ unchanged. In the honeycomb limit the bulk weights are given by $\rho_{4}=\rho_{5}=\rho_{6}=\rho_{7}=\rho_{8}=1, \rho_{2}=\rho_{3}=2 \cos (\lambda) \equiv t$ and $\rho_{1}=t^{2}$. Define the weights

$$
t_{\mathrm{O}} \equiv t=2 \cos (\lambda), \quad t_{\mathrm{Sp}} \equiv \cos (2 \lambda) \cos (\lambda)^{-1} .
$$

From Eqs. (2.23) we see that the remaining boundary weights are $\rho_{11}=t_{\mathrm{O}}$ and $\rho_{13}=t_{\mathrm{Sp}}$. An inspection of Figure 5 shows that we can achieve the same effect of the weight assignment $\rho_{i}$ by letting a honeycomb vertex (bulk or boundary) through which a bond runs carry a weight 1 , an "empty" bulk vertex a weight $t$, an empty left boundary vertex a weight $t_{\mathrm{O}}$ and an empty right boundary vertex a weight $t_{\mathrm{Sp}}$. This justifies the weight assignment (1.14) in the context of Figure 2, after a change of variables from $\lambda$ to $g$ related by

$$
\pi g=2 \pi-4 \lambda
$$

In the honeycomb limit, the diagonal-to-diagonal transfer matrix $t_{\mathrm{D}}(u)$ of Figure 3 becomes the transfer matrix $t_{\mathrm{H}}$ for taking a row of edges up the honeycomb strip of Figure 1 to the next similar row. The eigenvalue expression (2.19) then becomes (with a rescaling $u_{k} \rightarrow 2 u_{k}$ )

$$
\Lambda_{\mathrm{H}}=\prod_{j=1}^{M} \frac{\sinh \left(u_{j}+\frac{3}{2} \mathrm{i} \lambda\right) \sinh \left(u_{j}-\frac{3}{2} \mathrm{i} \lambda\right)}{\sinh \left(u_{j}+\frac{1}{2} \mathrm{i} \lambda\right) \sinh \left(u_{j}-\frac{1}{2} \mathrm{i} \lambda\right)}
$$




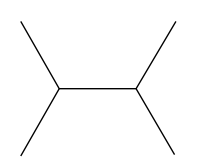

1

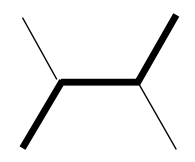

6

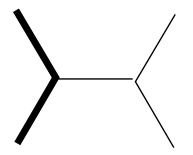

2

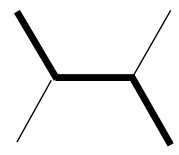

7

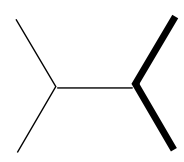

3

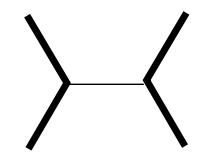

8

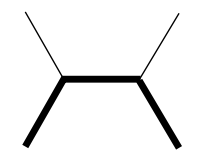

4
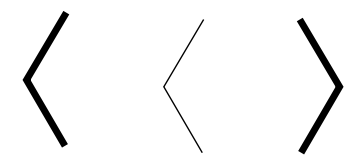

$\begin{array}{llll}10 & 11 & 12 & 13\end{array}$

Figure 5: The vertices of Figure 4 after the honeycomb limit has been taken.

The associated Bethe ansatz equations become $(k=1, \ldots, M)$

$$
\begin{aligned}
\Theta_{\mathrm{O}-\mathrm{Sp}}\left(u_{k}, \lambda\right)\left[\frac{\sinh \left(u_{k}-\frac{1}{2} \mathrm{i} \lambda\right) \sinh \left(u_{k}-\frac{3}{2} \mathrm{i} \lambda\right)}{\sinh \left(u_{k}+\frac{1}{2} \mathrm{i} \lambda\right) \sinh \left(u_{k}+\frac{3}{2} \mathrm{i} \lambda\right)}\right]^{N} \\
=\prod_{j \neq k}^{M} \frac{\sinh \left(u_{k}+u_{j}-2 \mathrm{i} \lambda\right) \sinh \left(u_{k}-u_{j}-2 \mathrm{i} \lambda\right)}{\sinh \left(u_{k}+u_{j}+2 \mathrm{i} \lambda\right) \sinh \left(u_{k}-u_{j}-2 \mathrm{i} \lambda\right)} \\
\quad \times \frac{\sinh \left(u_{k}+u_{j}+\mathrm{i} \lambda\right) \sinh \left(u_{k}-u_{j}+\mathrm{i} \lambda\right)}{\sinh \left(u_{k}+u_{j}-\mathrm{i} \lambda\right) \sinh \left(u_{k}-u_{j}-\mathrm{i} \lambda\right)},
\end{aligned}
$$

with the "boundary function" $\Theta_{\mathrm{O}-\mathrm{Sp}}(u, \lambda)$ defined as

$$
\Theta_{\mathrm{O}-\mathrm{Sp}}(u, \lambda)=\frac{\sinh (\mathrm{i} \lambda-2 u)}{\sinh (\mathrm{i} \lambda+2 u)} .
$$

In the language of Figure 2, the boundary weights for the O-Sp model are given by

$$
t_{l}=t_{\mathrm{O}}, \quad t_{r}=t_{\mathrm{Sp}} .
$$

The corresponding weights for the O-O model [19, 27] are

$$
t_{l}=t_{\mathrm{O}}, \quad t_{r}=t_{\mathrm{O}} .
$$

The transfer matrix eigenvalue expression (2.26) remains valid while the Bethe ansatz equations (2.27) have the boundary function $\Theta_{\mathrm{O}-\mathrm{Sp}}(u, \lambda)$ replaced by

$$
\Theta_{\mathrm{O}-\mathrm{O}}(u, \lambda)=\left[\frac{\sinh \left(u-\frac{1}{2} \mathrm{i} \lambda\right)}{\sinh \left(u+\frac{1}{2} \mathrm{i} \lambda\right)}\right]^{2} .
$$


For the Sp-Sp model [27] the corresponding weights are

$$
t_{l}=t_{\mathrm{Sp}}, \quad t_{r}=t_{\mathrm{Sp}}
$$

again with transfer matrix eigenvalue expression (2.26) and Bethe ansatz equations (2.27) with boundary function

$$
\Theta_{\mathrm{Sp}-\mathrm{Sp}}(u, \lambda)=\left[\frac{\cosh \left(u-\frac{1}{2} \mathrm{i} \lambda\right)}{\cosh \left(u+\frac{1}{2} \mathrm{i} \lambda\right)}\right]^{2}
$$

in place of $\Theta_{\mathrm{O}-\mathrm{Sp}}(u, \lambda)$.

Note the relation

$$
\Theta_{\mathrm{O}-\mathrm{Sp}}(u, \lambda)^{2}=\Theta_{\mathrm{O}-\mathrm{O}}(u, \lambda) \Theta_{\mathrm{Sp}-\mathrm{Sp}}(u, \lambda)
$$

between the various boundary functions. The choice of boundary weights - (2.29), (2.30) or (2.32) - and corresponding boundary function - (2.28), (2.31) or (2.33) - which arise from the appropriate combination of $K$-matrices governs the surface critical behaviour of the model. In the next section both universal and non-universal quantities will be derived from the eigenvalue expression (2.26) and Bethe ansatz equations (2.27) and their analogues for O-O and Sp-Sp boundaries.

We first solved the Bethe ansatz equations numerically and compared the eigenvalues so obtained to those from an exact numerical diagonalization of the transfer matrix for the vertex model (and also the loop model). The objective was twofold; firstly to confirm that the results obtained using the Doubling Hypothesis (2.9) are correct, and secondly to obtain the Bethe ansatz root distributions corresponding to various eigenstates of interest.

Restricting ourselves to even lattice sizes $N$, our chief findings can be summarised thus: Let $\ell=N-M$ label the sectors of the (vertex model) transfer matrix and $\Lambda_{\ell}$ be the leading eigenvalue in that sector. Then $\ell$ takes on values $0,1, \ldots, N-1$. For instance, $\Lambda_{0}$ is the largest eigenvalue in the ground state sector. For all cases except $\ell=0$ for Sp-Sp boundaries, the eigenvalue $\Lambda_{\ell}$ corresponds to all $N-\ell$ Bethe ansatz roots being real and strictly positive. For the dominant eigenvalue $\Lambda_{0}^{\mathrm{Sp}-\mathrm{Sp}}$ there are $N-1$ positive roots and a pure imaginary root at approximately $u_{1} \approx \mathrm{i}\left(\frac{\pi}{4}+\frac{\lambda}{2}\right)$. The scaling dimensions associated with $\Lambda_{\ell}$ are argued to give rise to the exponents characterizing the critical behaviour of the watermelon correlators. 


\section{Analysis of the Bethe ansatz equations}

At criticality, the free energy per site of the model is expected to scale with lattice size $N$ as (see, e.g. Ref. [21] and references therein)

$$
f_{N}=f_{\infty}+\frac{f_{S}^{(l)}}{N}+\frac{f_{S}^{(r)}}{N}-\frac{\pi \zeta c}{24 N^{2}}+O\left(N^{-3}\right),
$$

where $c$ is the central charge for the underlying conformal field theory and here $\zeta$ is a lattice-dependent scale factor. The bulk free energy $f_{\infty}$, the surface energies $f_{S}^{(l)}$ and $f_{S}^{(r)}$ associated with the left and right boundaries, and the central charge $c$ are all calculable from the Bethe ansatz equations, using a technique which is by now fairly standard (cf. [40, 11, 10, 11, 12, 19]). In particular, the calculation for O-O boundaries sketched in [19] is readily adaptable to handle the other two types of integrable boundaries.

We are mainly interested in the eigenvalues $\Lambda_{\mathrm{H}}$ corresponding to purely real Bethe ansatz roots. The eigenvalue expression (2.27) and Bethe ansatz equations (3.4) are invariant under $\lambda \rightarrow-\lambda$ and $\lambda \rightarrow \lambda+\pi$. Hence it is sufficient to consider $\lambda$ in the region $[0, \pi / 2]$. We restrict ourselves to values of $\lambda$ lying in the interval $(0, \pi / 2)$ to simplify certain Fourier integrals; the expressions for the central charges and scaling dimensions are nevertheless valid for the end-points, as can be shown using a limiting

procedure. The eigenvalue $\Lambda_{0}^{\mathrm{Sp}-\mathrm{Sp}}$ will be considered separately towards the end of the paper. Define the function

$$
\phi(u, \lambda)=2 \tan ^{-1}(\cot \lambda \tanh u)
$$

whose derivative with respect to $u$ is

$$
\phi^{\prime}(u, \lambda)=\frac{1}{i} \frac{\mathrm{d}}{\mathrm{d} u} \log \frac{\sinh (u-\mathrm{i} \lambda)}{\sinh (u+\mathrm{i} \lambda)}=\frac{2 \sin 2 \lambda}{\cosh 2 u-\cos 2 \lambda} .
$$

After taking logarithms, the Bethe ansatz equations (2.27) (and their analogues for the other boundary conditions) can be rewritten as

$$
\begin{aligned}
& N\left[\phi\left(u_{k}, \frac{1}{2} \lambda\right)+\phi\left(u_{k}, \frac{3}{2} \lambda\right)\right]+\phi_{\text {bound }}\left(u_{k}, \lambda\right)=2 \pi I_{k}+ \\
& \quad \sum_{j \neq k}^{M}\left[\phi\left(u_{k}+u_{j}, 2 \lambda\right)+\phi\left(u_{k}-u_{j}, 2 \lambda\right)-\phi\left(u_{k}+u_{j}, \lambda\right)-\phi\left(u_{k}-u_{j}, \lambda\right)\right] .
\end{aligned}
$$

Here the set of integers $I_{k}$ for $k=1, \ldots, M$ uniquely specifies an eigenstate of the transfer matrix. The term $\phi_{\text {bound }}(u, \lambda)$ depends on the boundary conditions chosen and is explicitly given by

$$
\phi_{\text {bound }}(u, \lambda)=\left\{\begin{array}{cc}
2 \phi\left(u, \frac{1}{2} \lambda\right) & \mathrm{O}-\mathrm{O} \\
\phi(2 u, \lambda) & \mathrm{O}-\mathrm{Sp} \\
2 \phi\left(u+\mathrm{i} \frac{\pi}{2}, \frac{1}{2} \lambda\right) & \mathrm{Sp}-\mathrm{Sp}
\end{array}\right.
$$


We determined the values of $I_{k}$ for the eigenvalues $\Lambda_{\ell}$ of interest numerically, i.e. by substituting the values of the Bethe ansatz roots $u_{j}$, determined from the numerical solution of the Bethe ansatz equations (2.27), into the logarithmic form (3.4). We expect that they can also be obtained by a careful consideration of the branch cuts involved in passing to the logarithmic form (see, e.g. [9]). The following identification is found for those eigenstates corresponding to real Bethe ansatz roots:

$$
\begin{aligned}
\Lambda_{\ell}^{\mathrm{O}-\mathrm{O}} & \leftrightarrow \quad I_{k}=k, \quad k=1, \ldots, M=N-\ell \\
\Lambda_{\ell}^{\mathrm{O}-\mathrm{Sp}} & \leftrightarrow \quad I_{k}=k, \quad k=1, \ldots, M=N-\ell \\
\Lambda_{\ell}^{\mathrm{Sp}-\mathrm{Sp}} & \leftrightarrow \quad I_{k}=k+1, \quad k=1, \ldots, M=N-\ell \quad(\ell \neq 0) .
\end{aligned}
$$

For the eigenvalue $\Lambda_{0}^{\mathrm{Sp}-\mathrm{Sp}}$ we find that $I_{j}=j$ for the real roots $j=2, \ldots, N$.

Define the "counting function" $z_{N}(u)$ by

$$
2 \pi z_{N}(u)=\phi\left(u, \frac{1}{2} \lambda\right)+\phi\left(u, \frac{3}{2} \lambda\right)-\frac{1}{N} \sum_{j=-M}^{M}\left[\phi\left(u-u_{j}, 2 \lambda\right)-\phi\left(u-u_{j}, \lambda\right)\right]+\frac{1}{N} \Phi(u),
$$

where

$$
\Phi(u)=\phi_{\text {bound }}(u, \lambda)+\phi(u, 2 \lambda)-\phi(u, \lambda)+\phi(2 u, 2 \lambda)-\phi(2 u, \lambda)
$$

contains the boundary contribution. For convenience, we have symmetrized $z_{N}(u)$ through the identification $u_{-k} \equiv-u_{k}$ for $k>0$ and $u_{0} \equiv 0$. Using the Bethe ansatz equations (3.4) it can be verified that for Bethe ansatz roots $u_{k}$ we have

$$
z_{N}\left(u_{k}\right)=\frac{I_{k}}{N}
$$

a property for which $z_{N}(u)$ is designed. Therefore, with the values of $I_{k}$ given in (3.6),

$$
\rho_{N}(u) \equiv \frac{\mathrm{d} z_{N}(u)}{\mathrm{d} u}
$$

becomes a root density for large $N$. Using this we can approximate for large $N$ (and therefore also $M$ )

$$
\frac{1}{N} \sum_{k=-M}^{M} f\left(u_{k}\right) \approx \int_{-\infty}^{\infty} \mathrm{d} v \rho_{\infty}(v) f(v)
$$

\subsection{Bulk free energy}

In the limit $N \rightarrow \infty$ Eq. (3.7) gives rise to the linear integral equation

$$
2 \pi \rho_{\infty}(u)=\phi^{\prime}\left(u, \frac{1}{2} \lambda\right)+\phi^{\prime}\left(u, \frac{3}{2} \lambda\right)-\int_{-\infty}^{\infty} \mathrm{d} v \rho_{\infty}(v)\left[\phi^{\prime}(u-v, 2 \lambda)-\phi^{\prime}(u-v, \lambda)\right]
$$


for the root density $\rho_{\infty}(u)$. This equation is readily solved by Fourier transforms. We use henceforth the notation

$$
\begin{aligned}
\widehat{a}(x) & =\frac{1}{2 \pi} \int_{-\infty}^{\infty} \mathrm{d} u e^{\mathrm{i} x u} a(u), \\
a(u) & =\int_{-\infty}^{\infty} \mathrm{d} x e^{-\mathrm{i} x u} \widehat{a}(x) .
\end{aligned}
$$

Using the result

$$
\widehat{\phi}^{\prime}(x, \lambda)=\frac{\sinh \left[\left(\frac{\pi}{2}-\lambda\right) x\right]}{\sinh \frac{1}{2} \pi x} \quad\left(0<\lambda<\frac{\pi}{2}\right),
$$

which can be established by contour integration, we find that the root density is given by

$$
2 \pi \widehat{\rho}_{\infty}(x)=\frac{2 \cosh \frac{1}{2} \lambda x}{2 \cosh \lambda x-1} .
$$

The eigenvalue expression (2.26) can be rewritten in the symmetrized form

$$
\Lambda_{\mathrm{H}}=\frac{\sinh \left(\frac{1}{2} \mathrm{i} \lambda\right)}{\sinh \left(\frac{3}{2} \mathrm{i} \lambda\right)} \prod_{j=-M}^{M} \frac{\sinh \left(u_{j}-\frac{3}{2} \mathrm{i} \lambda\right)}{\sinh \left(u_{j}-\frac{1}{2} \mathrm{i} \lambda\right)} .
$$

Let us denote for convenience

$$
\psi(u)=\log \frac{\sinh \left(u-\frac{3}{2} \mathrm{i} \lambda\right)}{\sinh \left(u-\frac{1}{2} \mathrm{i} \lambda\right)} .
$$

Then the free energy per site, defined as $f_{N} \equiv-\log \Lambda_{\mathrm{H}} / N$, becomes in the large $N$ limit

$$
\begin{aligned}
f_{\infty} & =-\int_{-\infty}^{\infty} \mathrm{d} v \rho_{\infty}(v) \psi(v) \\
& =-\int_{-\infty}^{\infty} \mathrm{d} x \frac{\sinh \left[\left(\frac{\pi}{2}-\lambda\right) x\right] \sinh (\lambda x)}{x \sinh \frac{1}{2} \pi x(2 \cosh \lambda x-1)}
\end{aligned}
$$

The last equality is obtained using (3.15) and the Fourier transform

$$
\widehat{\psi}(x)=\frac{e^{(\pi-2 \lambda) x / 2} \sinh \frac{1}{2} \lambda x}{x \sinh \frac{1}{2} \pi x} \quad\left(0<\lambda<\frac{2 \pi}{3}\right),
$$

which is also readily established by a contour integration involving its derivative. Not surprisingly, the boundary terms have not played a part up to this point. Indeed, the bulk free energy is the same if one starts from periodic boundary conditions [9]. In the next few sections we deal with the finite-size corrections in which the boundary terms are important. 


\subsection{Finite-size corrections}

Let us define now the functions

$$
\begin{aligned}
P_{N}(u) & =\rho_{N}(u)-\rho_{\infty}(u), \\
S_{N}(u) & =\frac{1}{N} \sum_{k=-M}^{M} \delta\left(u-u_{k}\right)-\rho_{N}(u), \\
K(u) & =\phi^{\prime}(u, 2 \lambda)-\phi^{\prime}(u, \lambda) .
\end{aligned}
$$

On differentiating (3.7) and using (3.12) we find that $P_{N}(u)$ satisfies the integral equation

$$
\begin{aligned}
P_{N}(u)+\frac{1}{2 \pi} \int_{-\infty}^{\infty} & \mathrm{d} v P_{N}(v) K(u-v) \\
& =-\frac{1}{2 \pi} \int_{-\infty}^{\infty} \mathrm{d} v K(u-v) S_{N}(v)+\frac{1}{2 \pi N} \Phi^{\prime}(u) .
\end{aligned}
$$

Taking the Fourier transform of both sides, gathering terms and then taking the inverse Fourier transform results in the following result for $P_{N}(u)$ :

$$
\begin{gathered}
P_{N}(u)=-\frac{1}{(2 \pi)^{2}} \int_{-\infty}^{\infty} \mathrm{d} w \int_{-\infty}^{\infty} \mathrm{d} v K(v-w) S_{N}(w) K_{1}(u-v) \\
+\frac{1}{(2 \pi)^{2} N} \int_{-\infty}^{\infty} \mathrm{d} v \Phi^{\prime}(v) K_{1}(u-v),
\end{gathered}
$$

where $K_{1}(u)$ is defined such that $\widehat{K}_{1}(x)=(1+\widehat{K}(x))^{-1}$. This can be given a different representation in terms of a function $K_{2}(u)$ defined such that $\widehat{K}_{2}(x)=-\widehat{K}(x) \widehat{K}_{1}(x)$ :

$$
P_{N}(u)=\frac{1}{2 \pi} \int_{-\infty}^{\infty} \mathrm{d} w S_{N}(w) K_{2}(u-w)+\frac{1}{(2 \pi)^{2} N} \int_{-\infty}^{\infty} \mathrm{d} v \Phi^{\prime}(v) K_{1}(u-v) .
$$

The Fourier transforms $\widehat{K}(x), \widehat{K}_{1}(x)$, and thus $\widehat{K}_{2}(x)$ follow from (3.14):

$$
\begin{aligned}
\widehat{K}(x) & =-\frac{2 \cosh \left[\frac{1}{2}(\pi-3 \lambda) x\right] \sinh \frac{1}{2} x \lambda}{\sinh \frac{1}{2} \pi x}, \\
\widehat{K}_{1}(x) & =\frac{\sinh \frac{1}{2} \pi x}{(2 \cosh \lambda x-1) \sinh \left[\left(\frac{\pi}{2}-\lambda\right) x\right]} .
\end{aligned}
$$

Analogous to Eq. (3.23) we find the following expression for the finite-size corrections to the free energy from the definitions of $f_{N}$ and $f_{\infty}$

$$
\begin{aligned}
f_{N}-f_{\infty} & =-\frac{1}{2 N} \log \left(\frac{1-\cos \lambda}{1-\cos 3 \lambda}\right)-\int_{-\infty}^{\infty} \mathrm{d} v P_{N}(v) \psi(v) \\
& -\int_{-\infty}^{\infty} \mathrm{d} v S_{N}(v) \psi(v) .
\end{aligned}
$$


Substituting in the result (3.25) for $P_{N}(u)$, we find that

$$
\begin{aligned}
f_{N}-f_{\infty} & =-\frac{1}{2 N} \log \left(\frac{1-\cos \lambda}{1-\cos 3 \lambda}\right)-\frac{1}{4 \pi^{2} N} \int_{-\infty}^{\infty} \mathrm{d} u \int_{-\infty}^{\infty} \mathrm{d} v \Phi^{\prime}(v) \psi(v) K_{1}(u-v) \\
& -\int_{-\infty}^{\infty} \mathrm{d} v S_{N}(v) e_{2}(v)
\end{aligned}
$$

where $e_{2}(u)$ is defined such that

$$
\widehat{e}_{2}(x)=\frac{\sinh \frac{1}{2} \lambda x}{x(2 \cosh \lambda x-1)} .
$$

In fact, by contour integration, this can be inverted to give $(\lambda>0)$

$$
e_{2}(u)=\frac{1}{2} \log \left(\frac{2 \cosh \frac{\pi u}{3 \lambda}+\sqrt{3}}{2 \cosh \frac{\pi u}{3 \lambda}-\sqrt{3}}\right) .
$$

Eqs. (3.25) and (3.28) are key equations in what follows and lead directly to the various terms in the free energy relation (3.1). Observe that in deriving these equations we have not had to specify in which sector of the transfer matrix we work. If we choose to be in sector $\ell$ then $f_{N}$ is to be interpreted as the lowest free energy in that sector. The scaling behaviour (3.1) in sector 0 leads to the central charge while that in sector $\ell$ leads to the relevant scaling dimensions.

\subsection{Surface free energies}

The $O(1 / N)$ terms in Eq. (3.28) give rise to the surface energy $f_{\mathrm{S}}$. Using the Fourier transform (3.14) we can evaluate $\widehat{\Phi^{\prime}}(x)$ for each type of boundary condition under consideration using the definitions (3.8) and (3.5). For O-O boundaries we recover a result of [19]

$$
\begin{aligned}
f_{\mathrm{S}}^{\mathrm{O}-\mathrm{O}} & =-\frac{1}{2} \log \left(\frac{1-\cos \lambda}{1-\cos 3 \lambda}\right) \\
& -4 \int_{-\infty}^{\infty} \mathrm{d} x \frac{\sinh \frac{\lambda x}{2} \cosh \frac{\lambda x}{4} \cosh \frac{(\pi-2 \lambda) x}{4} \sinh \frac{(\pi-3 \lambda) x}{4}\left(2 \cosh \frac{\lambda x}{2}-1\right)}{x \sinh \frac{\pi x}{2}(2 \cosh \lambda x-1)} .
\end{aligned}
$$

For O-Sp boundaries we find

$$
\begin{aligned}
f_{\mathrm{S}}^{\mathrm{O}-\mathrm{Sp}} & =-\frac{1}{2} \log \left(\frac{1-\cos \lambda}{1-\cos 3 \lambda}\right) \\
& -2 \int_{-\infty}^{\infty} \mathrm{d} x \frac{\sinh \frac{\lambda x}{2} \cosh \frac{(\pi-2 \lambda) x}{4} \sinh \frac{(\pi-6 \lambda) x}{4}}{x \sinh \frac{\pi x}{2}(2 \cosh \lambda x-1)} .
\end{aligned}
$$

\footnotetext{
${ }^{\dagger}$ There are misprints in the corresponding equations given in [19] for O-O boundaries.
} 
For Sp-Sp boundaries we use the Fourier transform

$$
\frac{1}{2 \pi} \int_{-\infty}^{\infty} \mathrm{d} u e^{\mathrm{i} x u} \phi^{\prime}\left(u+\mathrm{i} \frac{\pi}{2}, \frac{\lambda}{2}\right)=-\frac{\sinh \frac{1}{2} \lambda x}{\sinh \frac{1}{2} \pi x} \quad\left(0<\lambda<\frac{\pi}{2}\right),
$$

which can also be derived using contour integration, to obtain

$$
\begin{aligned}
f_{\mathrm{S}}^{\mathrm{Sp}-\mathrm{Sp}} & =-\frac{1}{2} \log \left(\frac{1-\cos \lambda}{1-\cos 3 \lambda}\right) \\
& +4 \int_{-\infty}^{\infty} \mathrm{d} x \frac{\sinh \frac{\lambda x}{2} \sinh \frac{\lambda x}{4} \cosh \frac{(\pi-3 \lambda) x}{4} \cosh \frac{(\pi-2 \lambda) x}{4}\left(1+2 \cosh \frac{\lambda x}{2}\right)}{x \sinh \frac{\pi x}{2}(2 \cosh \lambda x-1)}
\end{aligned}
$$

Non-trivial numerical checks can be performed at this stage. From numerical transfer matrix calculations we find that the largest eigenvalue $\Lambda_{0}$ of the transfer matrix at $\lambda=\pi / 8$ (i.e. the dilute polymer point $n=0$ ) is given by

$$
\Lambda_{0}=(2+\sqrt{2})^{N}\left\{\begin{array}{cc}
1 & \mathrm{O}-\mathrm{O} \\
(1+\sqrt{2})^{-1} & \mathrm{O}-\mathrm{Sp} \\
(1+\sqrt{2})^{-2} & \mathrm{Sp}-\mathrm{Sp}
\end{array}\right.
$$

without further corrections in $1 / N$. Therefore the surface free energies at $\lambda=\pi / 8$ are given respectively by $0, \log (1+\sqrt{2})$ and $2 \log (1+\sqrt{2})$. This is confirmed by numerical integration of Eqs. (3.31), (3.32) and (3.34).

It is not hard to show that the surface energies for general $\lambda$ are related by

$$
f_{\mathrm{S}}^{\mathrm{O}-\mathrm{Sp}}=\frac{1}{2}\left(f_{\mathrm{S}}^{\mathrm{O}-\mathrm{O}}+f_{\mathrm{S}}^{\mathrm{Sp}-\mathrm{Sp}}\right)
$$

which is, of course, a consequence of the relation (2.34) amongst the corresponding boundary functions. A comparison with Eq. (3.1) suggests sharing out the surface free energies in the following way:

$$
\begin{aligned}
f_{\mathrm{S}}^{(\mathrm{O})} & =\frac{1}{2} f_{\mathrm{S}-\mathrm{O}}^{\mathrm{O}} \\
f_{\mathrm{S}}^{(\mathrm{S})} & =\frac{1}{2} f_{\mathrm{S}}^{\mathrm{Sp}-\mathrm{Sp}} .
\end{aligned}
$$

\subsection{Wiener-Hopf integral equation}

We move on now to the next leading order in $1 / N$ in the finite-size correction $f_{N}-f_{\infty}$. The Euler-Maclaurin formula gives rise to

$$
\begin{aligned}
\int_{-\infty}^{\infty} \mathrm{d} u g(u) S_{N}(u) \approx & -\left(\int_{-\infty}^{-\Lambda} \mathrm{d} u+\int_{\Lambda}^{\infty} \mathrm{d} u\right) g(u) \rho_{N}(u)+ \\
& \frac{1}{2 N}(g(\Lambda)+g(-\Lambda))+\frac{1}{12 N^{2} \rho_{N}(\Lambda)}\left(g^{\prime}(\Lambda)+g^{\prime}(-\Lambda)\right)
\end{aligned}
$$


for arbitrary $g(u)$ analytic in $[-\Lambda, \Lambda]$ where $\Lambda$ is the largest root. In other words, $\Lambda$ is such that $z_{N}(\Lambda)=I_{\max } / N$ where, on using (3.6), $I_{\max }=N-\ell$ in sector $\ell$ for O-O and O-Sp boundaries, and $I_{\max }=N-\ell+1$ in sector $\ell \neq 0$ for Sp-Sp boundaries. The condition on $\Lambda$ leads to the sum rulef

$$
\begin{aligned}
\int_{\Lambda}^{\infty} \mathrm{d} u \rho_{N}(u) & =z_{N}(\infty)-z_{N}(\Lambda) \\
& =1+\frac{2 \lambda}{\pi}\left(\frac{M}{N}-1\right)-\frac{\lambda}{\pi N}+\frac{\phi_{\text {bound }}(\infty, \lambda)}{2 \pi N}-\frac{I_{\max }}{N},
\end{aligned}
$$

where the last equality follows from the definition (3.7) and the result $\phi(\infty, \lambda)=\pi-2 \lambda$. Applying (3.38) to the first integral of Eq. (3.25) we obtain

$$
\begin{aligned}
\rho_{N}(u)-\rho_{\infty}(u) \approx & \frac{1}{4 \pi^{2} N} \int_{-\infty}^{\infty} \mathrm{d} v K_{1}(u-v) \Phi^{\prime}(v)-\frac{1}{2 \pi} \int_{\Lambda}^{\infty} \mathrm{d} v \rho_{N}(v) K_{2}(u-v)+ \\
& \frac{1}{4 \pi N} K_{2}(u-\Lambda)-\frac{1}{24 \pi N^{2} \rho_{N}(\Lambda)} K_{2}^{\prime}(u-\Lambda),
\end{aligned}
$$

which is valid for $u \geq \Lambda$. Under this condition, certain terms implied by (3.38) are negligible because $K_{2}(u)$ decreases exponentially with increasing $u$ and have been dropped. Applying (3.38) to the last term of Eq. (3.28) and using the fact that $e_{2}(u)$ is an even function we obtain also

$$
-\int_{-\infty}^{\infty} \mathrm{d} v S_{N}(v) e_{2}(v) \approx 2 \int_{\Lambda}^{\infty} \mathrm{d} u \rho_{N}(u) e_{2}(u)-\frac{1}{N} e_{2}(\Lambda)-\frac{e_{2}^{\prime}(\Lambda)}{6 N^{2} \rho_{N}(\Lambda)} .
$$

This term describes the $O\left(1 / N^{2}\right)$ correction to the free energy and in the thermodynamic limit is governed by the behaviour of $\rho_{N}(\Lambda)$ for large $\Lambda$. This in turn can be found by solving Eq. (3.40) subject to the sum rule (3.39), as we now demonstrate.

Define the functions

$$
\begin{aligned}
\chi(u) & =\rho_{N}(u+\Lambda), \\
f(u) & =\rho_{\infty}(u+\Lambda), \\
\epsilon(u) & =\frac{1}{4 \pi^{2} N} \int_{-\infty}^{\infty} \mathrm{d} v K_{1}(u+\Lambda-v) \Phi^{\prime}(v) .
\end{aligned}
$$

The integral equation (3.40) can then be rewritten as

$$
\chi(u) \approx f(u)+\epsilon(u)-\frac{1}{2 \pi} \int_{0}^{\infty} \mathrm{d} v \chi(v) K_{2}(u-v)+\frac{1}{4 \pi N} K_{2}(u)-\frac{K_{2}^{\prime}(u)}{24 \pi N^{2} \rho_{N}(\Lambda)} .
$$

This can be recognised as a Wiener-Hopf type integral equation for $\chi(u)$ which can be solved by standard means.

\footnotetext{
竬位 corrects a misprint in the sum rule for O-O boundaries given in 19
} 
First split $\widehat{\chi}(x)=\widehat{\chi}_{+}(x)+\widehat{\chi}_{-}(x)$ into components analytic in the upper and lower half-planes $\Pi_{ \pm}$, with

$$
\widehat{\chi}_{+}(x)=\frac{1}{2 \pi} \int_{0}^{\infty} \mathrm{d} u e^{\mathrm{i} x u} \chi(u) .
$$

Then the Fourier transform of the integral equation (3.43) can be written as

$$
\widehat{\chi}(x) \approx \widehat{f}(x)+\widehat{\epsilon}(x)-\widehat{\chi}_{+}(x) \widehat{K}_{2}(x)+\frac{1}{2 \pi} \widehat{K}_{2}(x) C(x),
$$

where we define

$$
C(x) \equiv \frac{1}{2 N}+\frac{\mathrm{i} x}{12 N^{2} \rho_{N}(\Lambda)} .
$$

Let $F(x) \equiv \widehat{f}(x)+\widehat{\epsilon}(x)$ be split into components analogous to $\widehat{\chi}(x)$. Then (3.45) can be rewritten as

$$
\widehat{\chi}_{-}(x)+\left[1+\widehat{K}_{2}(x)\right]\left[\widehat{\chi}_{+}(x)-\frac{1}{2 \pi} C(x)\right] \approx F_{+}(x)+F_{-}(x)-\frac{1}{2 \pi} C(x) .
$$

Now, if we can factorize

$$
\left[1+\widehat{K}_{2}(x)\right]^{-1}=G_{+}(x) G_{-}(x),
$$

where $G_{+}(x)\left(G_{-}(x)\right)$ is analytic in $\Pi_{+}$(respectively, $\left.\Pi_{-}\right)$, and $G_{-}(x) F_{+}(x)$ can be split into + and - components

$$
G_{-}(x) F_{+}(x)=Q_{+}(x)+Q_{-}(x),
$$

then Eq. (3.47) can be written in the form

$$
\begin{aligned}
\frac{\hat{\chi}_{+}(x)-\frac{1}{2 \pi} C(x)}{G_{+}(x)}-Q_{+}(x) & =Q_{-}(x)-G_{-}(x)\left[\widehat{\chi}_{-}(x)+\frac{1}{2 \pi} C(x)-F_{-}(x)\right] \\
& \equiv P(x) .
\end{aligned}
$$

Since the first two terms are each analytic in $\Pi_{+}$and $\Pi_{-}$respectively, $P(x)$ must be a regular function and can be determined, for instance, from the large $x$ behaviour of the first term.

Let us now confirm the decompositions (3.48) and (3.49). Firstly, we find from (3.26) that

$$
\left[1+\widehat{K}_{2}(x)\right]^{-1}=\frac{(2 \cosh \lambda x-1) \sinh [(\pi-2 \lambda) x / 2]}{\sinh (\pi x / 2)} .
$$

It indeed factorizes as (3.48) with 10, 11

$$
\begin{aligned}
G_{+}(x) & =\frac{2 \sqrt{\pi(\pi-2 \lambda)} \Gamma\left(1-\mathrm{i} \frac{x}{2}\right) e^{h(x)}}{\Gamma\left(1-\frac{\mathrm{i} x}{2 \pi}(\pi-2 \lambda)\right) \Gamma\left(\frac{1}{6}-\frac{\mathrm{i} \lambda}{2 \pi} x\right) \Gamma\left(\frac{5}{6}-\frac{\mathrm{i} \lambda}{2 \pi} x\right)} \\
& =G_{-}(-x),
\end{aligned}
$$


where

$$
h(x)=\frac{\mathrm{i} x}{2}\left[\log \frac{\pi}{\lambda}-\frac{(\pi-2 \lambda)}{\pi} \log \frac{\pi-2 \lambda}{\lambda}\right] .
$$

As $|x| \rightarrow \infty$ in $\Pi_{+}$we find, on using Stirling's formula, that

$$
G_{+}(x) \sim 1+\frac{g_{1}}{x}+\frac{g_{2}}{x^{2}}+O\left(x^{-3}\right),
$$

where the coefficients are given by

$$
\begin{aligned}
& g_{1}=\frac{\mathrm{i}}{3}\left[\frac{1}{2}-\frac{\pi}{2(\pi-2 \lambda)}-\frac{\pi}{6 \lambda}\right], \\
& g_{2}=\frac{1}{2} g_{1}^{2} .
\end{aligned}
$$

Next, from the definition (3.42) and the result (3.15) we have

$$
\widehat{f}(x)=\frac{e^{-\mathrm{i} x \Lambda}}{2 \pi} \frac{2 \cosh (\lambda x / 2)}{(2 \cosh \lambda x-1)},
$$

whose leading pole in $\Pi_{-}$is at $x=-\mathrm{i} \pi / 3 \lambda$. Keeping only this leading pole term we obtain

$$
\widehat{f}_{+}(x) \approx \frac{e^{-\mathrm{i} x \Lambda}}{2 \pi} \frac{2 \sqrt{3} \cosh (\lambda x / 2)}{(\pi-3 \mathrm{i} \lambda x)} \approx F_{+}(x) .
$$

Here we have ignored the contribution to $F(x)$ from $\epsilon(x)$ (cf. Eq. (3.42)), which contributes to the finite-size corrections at a higher order in $1 / N$. One can now see that $G_{-}(x) F_{+}(x)$ has only one pole in $\Pi_{-}$, at $x=-\mathrm{i} \pi / 3 \lambda$ and use this fact to obtain

$$
Q_{+}(x)=\frac{3 G_{+}\left(\frac{\mathrm{i} \pi}{3 \lambda}\right) e^{-\frac{\pi \Lambda}{3 \lambda}}}{2 \pi(\pi-3 \mathrm{i} \lambda x)} .
$$

In particular, $Q_{+}(x) \rightarrow 0$ as $|x| \rightarrow \infty$ in $\Pi_{+}$. In the same limit, $\widehat{\chi}_{+}(x) \rightarrow 0$ from the requirement that $\chi_{+}(u)$ is integrable at the origin. Therefore from Eqs. (3.50), (3.46) and (3.54) we obtain

$$
P(x)=-\frac{1}{2 \pi}\left[\frac{1}{2 N}+\frac{\mathrm{i}\left(x-g_{1}\right)}{12 N^{2} \rho_{N}(\Lambda)}\right] .
$$

Knowing $C(x), G_{+}(x), Q_{+}(x)$ and $P(x)$, we are then in a position to obtain $\widehat{\chi}_{+}(x)$ using Eq. (3.50). We now note from the definitions (3.42) and (3.44) that $\hat{\chi}_{+}(x)=$ $\frac{1}{2 \pi} \int_{0}^{\infty} \mathrm{d} u e^{\mathrm{i} x u} \rho_{N}(u+\Lambda)$. Therefore from Eq. (3.50) we obtain

$$
\frac{e^{-\mathrm{i} x \Lambda}}{2 \pi} \int_{\Lambda}^{\infty} \mathrm{d} u e^{\mathrm{i} x u} \rho_{N}(u)=G_{+}(x)\left[P(x)+Q_{+}(x)\right]+\frac{1}{2 \pi} C(x) .
$$

From this equation, two key results (3.64) and (3.67) will be obtained. We note at this point that since we have dropped $\widehat{\epsilon}(x)$ - which contains the contribution from 
$\Phi(u)$ and therefore $\phi_{\text {bound }}(u)$ - from $F(x)$, Eq. (3.60) does not depend on the boundary conditions.

Taking the inverse Fourier transform of $\widehat{\chi}_{+}(x)$, we obtain $\rho_{N}(\Lambda)$ as a contour integral, which can be written as a residue at infinity:

$$
\chi_{+}(0)=\frac{1}{2} \rho_{N}(\Lambda)=-\mathrm{i} \pi \lim _{x \rightarrow \infty}\left[x G_{+}(x)\left[P(x)+Q_{+}(x)\right]+\frac{x}{2 \pi} C(x)\right] .
$$

We now subsititute Eqs. (3.46), (3.59), (3.54) and (3.58) into (3.61) and evaluate the limit to find

$$
\begin{aligned}
N \rho_{N}(\Lambda)= & \frac{\mathrm{i} g_{1}}{2}+\frac{g_{1}^{2}-g_{2}}{12 N \rho_{N}(\Lambda)}+\frac{2 \pi^{2} N}{3 \lambda}\left[Q_{+}(0)+P(0)\right]+ \\
& \frac{\pi}{3 \lambda}\left[\frac{1}{2}-\frac{\mathrm{i} g_{1}}{12 N \rho_{N}(\Lambda)}\right] .
\end{aligned}
$$

In arriving at the final result we have eliminated $G_{+}(\mathrm{i} \pi / 3 \lambda)$ in favour of $Q_{+}(0)$ using (3.58). Define

$$
\rho \equiv N \rho_{N}(\Lambda), \quad \alpha \equiv N\left[Q_{+}(0)+P(0)\right]
$$

Then Eq. (3.62) can be succintly written as a quadratic equation for $\rho$

$$
\rho^{2}=\rho\left(\frac{\mathrm{i} g_{1}}{2}+\frac{2 \pi^{2}}{3 \lambda} \alpha+\frac{\pi}{6 \lambda}\right)+\frac{g_{1}^{2}-g_{2}}{12}-\frac{\mathrm{i} \pi g_{1}}{36 \lambda} .
$$

We will see in the next section that the $O\left(N^{-2}\right)$ correction to the free energy can be expressed in terms of $\rho, \alpha$ and $g_{1}$. We will also see that on using the quadratic equation (3.64) this expression becomes just a function of $\alpha^{2}$.

Let us now relate the evaluation of $\alpha$ to the sum rule (3.39). On setting $x=0$ in Eq. (3.60) we obtain

$$
\int_{\Lambda}^{\infty} \mathrm{d} u \rho_{N}(u)=2 \pi G_{+}(0)\left[P(0)+Q_{+}(0)\right]+C(0) .
$$

From 3.52 we have

$$
G_{+}(0)=\frac{2 \sqrt{\pi(\pi-2 \lambda)}}{\Gamma\left(\frac{1}{6}\right) \Gamma\left(\frac{5}{6}\right)}=\frac{\sqrt{\pi(\pi-2 \lambda)}}{\pi},
$$

and from (3.46) we can evaluate $C(0)$. A comparison of (3.65) with the sum rule 3.39$)$ then leads to the result

$$
\begin{aligned}
& 4 \pi^{2} \alpha^{2}= \\
& \quad\left(1-\frac{2 \lambda}{\pi}\right)^{-1}\left[N-\frac{1}{2}+\frac{2 \lambda}{\pi}(M-N)-\frac{\lambda}{\pi}+\frac{\phi_{\text {bound }}(\infty, \lambda)}{2 \pi}-I_{\max }\right]^{2},
\end{aligned}
$$

where the boundary contribution has finally reappeared. 


\subsection{Central charge and scaling dimensions}

We are now ready to return to the finite-size correction to the free energy (3.41). From Eq. (3.30) we have

$$
e_{2}(u) \sim \sqrt{3} e^{-\frac{\pi u}{3 \lambda}}
$$

for large $u$. Substitution into Eq. (3.41) yields

$$
\begin{aligned}
& -\int_{-\infty}^{\infty} \mathrm{d} v S_{N}(v) e_{2}(v) \approx \\
& \quad 2 \sqrt{3} \int_{\Lambda}^{\infty} \mathrm{d} u e^{\mathrm{i} u\left(\frac{\mathrm{i} \pi}{3 \lambda}\right)} \rho_{N}(u)-\left[\frac{\sqrt{3}}{N}-\frac{\sqrt{3} \pi}{18 \lambda N^{2} \rho_{N}(\Lambda)}\right] e^{-\frac{\pi \Lambda}{3 \lambda}} .
\end{aligned}
$$

Using the equation (3.60) with $x=\mathrm{i} \pi / 3 \lambda$ and the expression (3.46) for $C(x)$ we end up with

$$
-\int_{-\infty}^{\infty} \mathrm{d} v S_{N}(v) e_{2}(v) \approx 4 \sqrt{3} \pi e^{-\frac{\pi \Lambda}{3 \lambda}} G_{+}\left(\frac{\mathrm{i} \pi}{3 \lambda}\right)\left[P\left(\frac{\mathrm{i} \pi}{3 \lambda}\right)+Q_{+}\left(\frac{\mathrm{i} \pi}{3 \lambda}\right)\right] .
$$

Both $G_{+}\left(\frac{\mathrm{i} \pi}{3 \lambda}\right)$ and $Q_{+}\left(\frac{\mathrm{i} \pi}{3 \lambda}\right)$ can be eliminated in favour of $Q_{+}(0)$ using (3.58). This in turn can be expressed in terms of $\alpha$ and $\rho$. We have thus

$$
\begin{aligned}
-\int_{-\infty}^{\infty} \mathrm{d} v S_{N}(v) e_{2}(v) & \approx \frac{4 \sqrt{3} \pi}{N^{2}}\left[\frac{2 \pi^{2}}{3} \alpha+\frac{\pi}{3}\left(\frac{1}{2}-\frac{\mathrm{i} g_{1}}{12 \rho}\right)\right] \\
& \times\left(\frac{\alpha}{2}-\frac{1}{8 \pi}+\frac{\mathrm{i} g_{1}}{48 \pi \rho}+\frac{1}{72 \lambda \rho}\right) .
\end{aligned}
$$

Expanding out the terms and using the quadratic equation (3.64) for $\rho$ we find eventually that

$$
-\int_{-\infty}^{\infty} \mathrm{d} v S_{N}(v) e_{2}(v) \approx-\frac{\pi}{24 N^{2}}\left(\frac{2}{\sqrt{3}}\right)\left(1-48 \pi^{2} \alpha^{2}\right) .
$$

Now from Eq. (3.67) we have the result

$$
4 \pi^{2} \alpha^{2}=\left(1-\frac{2 \lambda}{\pi}\right)^{-1}\left\{\begin{array}{cc}
{\left[\ell\left(1-\frac{2 \lambda}{\pi}\right)-\frac{2 \lambda}{\pi}+\frac{1}{2}\right]^{2}} & \mathrm{O}-\mathrm{O} \\
{\left[\ell\left(1-\frac{2 \lambda}{\pi}\right)-\frac{2 \lambda}{\pi}\right]^{2}} & \mathrm{O}-\mathrm{Sp} \\
{\left[\ell\left(1-\frac{2 \lambda}{\pi}\right)-\frac{2 \lambda}{\pi}-\frac{1}{2}\right]^{2}} & \mathrm{Sp}-\mathrm{Sp} \quad(\ell \neq 0)
\end{array}\right.
$$

for the various eigenstates of interest, except for the ground state with Sp-Sp boundary conditions. Let us first consider the ground states $(\ell=0)$ for the O-O and O-Sp cases. Substituting the result for $\alpha^{2}$ in Eq. (3.73) into Eq. (3.72) and comparing with the result (3.1) leads to the identification $\zeta=2 / \sqrt{3}$ for the scale factore, together with the

\footnotetext{
$\S$ This corrects misprints in [19, 28]
} 
central charges

$$
\begin{aligned}
c^{\mathrm{O}-\mathrm{O}} & =1-\frac{3(\pi-4 \lambda)^{2}}{\pi(\pi-2 \lambda)} \\
c^{\mathrm{O}-\mathrm{Sp}} & =1-\frac{3(4 \lambda)^{2}}{\pi(\pi-2 \lambda)}
\end{aligned}
$$

With the change of variables from $\lambda$ to $g$ related by (2.25), we obtain the results (1.8) and (1.15). The mixed-boundary scaling index $t^{\mathrm{O}-\mathrm{Sp}}$ defined by

$$
c^{\mathrm{O}-\mathrm{Sp}}=c^{\mathrm{O}-\mathrm{O}}-24 t^{\mathrm{O}-\mathrm{Sp}}
$$

is therefore given by $t^{\mathrm{O}-\mathrm{Sp}}=(3-2 g) / 4 g=h_{1,2}$ in the Kac formula (1.10). Using the notation $c_{\ell}$ for the effective central charge for the eigenstate labelled by $\ell$, we find from (3.72) and (3.73) that

$$
\begin{aligned}
c_{\ell}^{\mathrm{O}-\mathrm{O}}-c^{\mathrm{O}-\mathrm{O}} & =-24\left[\left(\frac{1}{2}-\frac{2 \lambda}{\pi}\right) \ell+\left(\frac{1}{2}-\frac{\lambda}{\pi}\right) \ell^{2}\right], \\
c_{\ell}^{\mathrm{O}-\mathrm{Sp}}-c^{\mathrm{O}-\mathrm{Sp}} & =-24\left[-\frac{2 \lambda}{\pi} \ell+\left(\frac{1}{2}-\frac{\lambda}{\pi}\right) \ell^{2}\right] .
\end{aligned}
$$

The scaling dimensions $X_{\ell}$ defined through the inverse correlation lengths 42]

$$
\xi_{\ell}^{-1}=\log \frac{\Lambda_{0}}{\Lambda_{\ell}} \sim \frac{\pi \zeta X_{\ell}}{N}
$$

are then given by $(1.9)$ and (1.16).

Let us now turn to the ground state for Sp-Sp boundaries, where there is an imaginary Bethe ansatz root and the preceding analysis has to be slightly modified. Firstly we noted numerically that the position of the imaginary root reaches its asymptotic value much faster than $1 / N$. We therefore consider it to be fixed; and that this assumption does not affect the finite-size corrections to the order in which we are interested. For convenience we perform a shift $u_{j} \rightarrow u_{j-1}$ in the eigenvalue expression (2.26) and associated Bethe ansatz equations. The imaginary root is now $u_{0} \approx \mathrm{i}\left(\frac{\pi}{4}+\frac{\lambda}{2}\right)$ while the eigenvalue expression becomes

$$
\Lambda_{0}^{\mathrm{Sp}-\mathrm{Sp}}=\frac{\sinh \left(u_{0}+\frac{3}{2} \mathrm{i} \lambda\right) \sinh \left(u_{0}-\frac{3}{2} \mathrm{i} \lambda\right)}{\sinh \left(u_{0}+\frac{1}{2} \mathrm{i} \lambda\right) \sinh \left(u_{0}-\frac{1}{2} \mathrm{i} \lambda\right)} \prod_{j=1}^{N-1} \frac{\sinh \left(u_{j}+\frac{3}{2} \mathrm{i} \lambda\right) \sinh \left(u_{j}-\frac{3}{2} \mathrm{i} \lambda\right)}{\sinh \left(u_{j}+\frac{1}{2} \mathrm{i} \lambda\right) \sinh \left(u_{j}-\frac{1}{2} \mathrm{i} \lambda\right)} .
$$

The Bethe ansatz equations (3.4) are now valid for $k=0,1, \ldots, M=N-1$, with $I_{k}=k+1$. The product term in (3.80), which involves only real roots $u_{j}$ can now be treated as before. The only difference is that the counting function $z_{N}(u)$ has to be modified in order that $z_{N}\left(u_{k}\right)=I_{k} / N$ holds for the real roots $u_{k}$ in the shifted variables. We find that $\Phi(u)$ in (3.7) has to be replaced by

$$
\begin{aligned}
\Phi_{0}^{\mathrm{Sp}-\mathrm{Sp}}(u) & =\phi_{\text {bound }}(u, \lambda)-\phi\left(u+u_{0}, 2 \lambda\right)+\phi\left(u+u_{0}, \lambda\right) \\
& +\phi(2 u, 2 \lambda)-\phi(2 u, \lambda)
\end{aligned}
$$


where unlike previously $u_{0}$ is no longer identified with 0 . With these changes, the analysis of finite-size corrections then proceeds as before. Since we have already obtained the surface energy from the excitations, we restrict ourselves to the $O\left(N^{-2}\right)$ terms. We find that Eq. (3.72) is unchanged. The sum rule (3.39) on the other hand is now

$$
\int_{\Lambda}^{\infty} \mathrm{d} u \rho_{N}(u)=\frac{1}{N}-\frac{2 \lambda}{\pi N}
$$

due to $\Phi_{0}^{\mathrm{Sp}-\mathrm{Sp}}(u)$. This results in the replacement of (3.73) with

$$
4 \pi^{2} \alpha^{2}=\left(1-\frac{2 \lambda}{\pi}\right)^{-1}\left(\frac{1}{2}-\frac{2 \lambda}{\pi}\right)^{2} .
$$

Equations (3.72) and (3.83) then give rise to

$$
c^{\mathrm{Sp}-\mathrm{Sp}}=1-\frac{3(\pi-4 \lambda)^{2}}{\pi(\pi-2 \lambda)},
$$

which is the same as for O-O boundaries. From Eqs. (3.72) and (3.73) we can now calculate the effective central charge for $\Lambda_{\ell}^{\mathrm{Sp}-\mathrm{Sp}}$ :

$$
c_{\ell}^{\mathrm{Sp}-\mathrm{Sp}}-c^{\mathrm{Sp}-\mathrm{Sp}}=-24\left[\frac{2 \lambda}{\pi}\left(1-\frac{2 \lambda}{\pi}\right)^{-1}+\left(-\frac{1}{2}-\frac{2 \lambda}{\pi}\right) \ell+\left(\frac{1}{2}-\frac{\lambda}{\pi}\right) \ell^{2}\right] .
$$

The scaling dimensions $X_{\ell}^{\mathrm{Sp}-\mathrm{Sp}}$ are then given by (1.13).

\section{Discussion}

In this paper we have studied the $O(n)$ loop model on the honeycomb lattice with open boundaries from the point of view of the underlying Izergin-Korepin $R$-matrix and its associated $K$-matrices. Three inequivalent sets of integrable boundary weights and their corresponding Bethe ansatz solutions were thus obtained. Two of these sets were of "pure-type" 27 and one of mixed-type. One of the pure-type boundary weights (denoted here by $\mathrm{O}-\mathrm{O}$ ), with the bulk and surface couplings being the same, was previously studied using the coordinate Bethe ansatz [19] and argued to correspond to the $O(n)$ model at the ordinary surface transition. By analysing the associated Bethe ansatz equations for the second set of pure boundary weights (denoted here by $\mathrm{Sp}-\mathrm{Sp}$ ) we found agreement with known results (some of which were conjectures) for the special surface transition [15, 20], which in the $n \rightarrow 0$ limit corresponds to the adsorption transition for polymers. This justifies our claim to have found the critical couplings for the $O(n)$ loop model on the honeycomb lattice at the special transition and allows us to "prove" the abovementioned conjectures. The set of mixed-type boundary weights therefore should correspond to the $O(n)$ model at the mixed ordinary-special surface 
transition. Our Bethe ansatz analysis gives a central charge and mixed-boundary scaling index which are indeed in agreement with a recent result [21] for this transition, providing further evidence for our claim. In addition our analysis has furnished the scaling dimensions for this transition.

It is interesting that the $K$-matrices for the $A_{2}^{(2)}$ model have "accounted for" the ordinary and special surface transitions in the manner described in this paper. This leaves the interesting open problem of finding an integrable lattice $O(n)$ model which exhibits the extraordinary surface transition [26] which corresponds to having fixed $O(n)$ spins at the boundary, and for which conformal invariance predictions exist [15, 21. It is also an interesting open problem to derive the physical boundary $S$-matrices, which constitute a starting point for the techniques of [20], from the $K$-matrices. This ought to be achievable using the Bethe ansatz analysis performed here, along the lines of [43] for the XXX model, or using the vertex operator approach 44.

\section{Acknowledgements}

This work has been supported by the Australian Research Council.

\section{References}

[1] H. E. Stanley, Phys. Rev. Lett. 20 (1968) 589.

[2] E. Domany, D. Mukamel, B. Nienhuis and A. Schwimmer, Nucl. Phys. B 190 (1981) 279

[3] B. Nienhuis, Phys. Rev. Lett. 49 (1982) 1062.

[4] B. Nienhuis, in Phase Transitions and Critical Phenomena Vol 11, ed. C. Domb and J. Lebowitz, Academic Press, London, 1987.

[5] J. L. Cardy, Nucl. Phys. B 240 (1984) 514.

[6] B. Duplantier and H. Saleur, Phys. Rev. Lett. 57 (1986) 3179.

[7] J. L. Cardy, in Phase Transitions and Critical Phenomena Vol 11, ed. C. Domb and J. Lebowitz, Academic Press, London, 1987.

[8] B. Duplantier, Phys. Rep. 184 (1989) 229.

[9] R. J. Baxter, J. Phys. A 19 (1986) 2821.

[10] M. T. Batchelor and H W J Blöte, Phys. Rev. Lett. 61 (1988) 138; Phys. Rev. B 39 (1989) 2391.

[11] J. Suzuki, J. Phys. Soc. Japan 57 (1988) 2966.

[12] J. Suzuki, T. Nagao and M. Wadati, Int. J. Mod. Phys. B 6 (1992) 1119.

[13] T. W. Burkhardt and J. L. Cardy, J. Phys. A 20 (1987) L233. 
[14] H. W. J. Blöte and B. Nienhuis, J. Phys. A 22 (1989) 1415.

[15] T. W. Burkhardt, E. Eisenriegler and I. Guim, Nucl. Phys. B 316 (1989) 559.

[16] A. B. Zamolodchikov, Mod. Phys. Lett. A 6 (1991) 1807

[17] P. Fendley, H. Saleur and Al. B. Zamolodchikov, Int. J. Mod. Phys. A 8 (1993) 5717.

[18] J. Cardy and G. Mussardo, Nucl. Phys. B 410 (1993) 451.

[19] M. T. Batchelor and J. Suzuki, J. Phys. A 26 (1993) L729.

[20] P. Fendley and H. Saleur, J. Phys. A 27 (1994) L789.

[21] T. W. Burkhardt and E. Eisenriegler, Nucl. Phys. B 424 (1994) 487.

[22] P. G. de Gennes, Scaling concepts in polymer physics, Cornell University, 1979.

[23] K. De'Bell and T. Lookman, Rev. Mod. Phys. 65 (1993) 87.

[24] I. Guim and T. W. Burkhardt, J. Phys. A 22 (1989) 1131.

[25] I. Guim and T. W. Burkhardt, Phys. Rev. E 49 (1994) 1495.

[26] K. Binder, in Phase transitions and critical phenomena, vol. 8, ed. C. Domb and J. L. Lebowitz (Academic Press, New York, 1983).

[27] C. M. Yung and M. T. Batchelor, Nucl. Phys. B 435 (1995) 430.

[28] M. T. Batchelor and C. M. Yung, Phys. Rev. Lett. 74 (1995) 2026.

[29] E. K. Sklyanin, J. Phys. A 21 (1988) 2375.

[30] L. Mezincescu and R. I. Nepomechie, J. Phys. A 24 (1991) L17.

[31] C. Destri and H. J. de Vega, Nucl. Phys. B374 (1992) 692.

[32] A. G. Izergin and V. E. Korepin, Commun. Math. Phys. 79 (1981) 303.

[33] B. Nienhuis, Int. Jour. Mod. Phys. B 4 (1990) 929.

[34] N. Yu. Reshetikhin, J. Phys. A 24 (1991) 2387.

[35] L. Mezincescu and R. I. Nepomechie, Int. Jour. Mod. Phys. A 7 (1991) 5231.

[36] M. T. Batchelor and C. M. Yung, "Surface critical behaviour of the honeycomb $O(n)$ loop model with mixed ordinary and special boundary conditions", ANU preprint.

[37] S. Artz, L. Mezincescu and R.I. Nepomechie, "Spectrum of transfer matrix for $U_{q}\left(B_{n}\right)$-invariant $A_{2 n-1}^{(2)}$ open spin chain", hep-th/9409130, Int. J. Mod. Phys. A, in press. 
[38] C.M. Yung and M.T. Batchelor, Phys. Lett. A198 (1995) 395.

[39] L. Mezincescu and R. I. Nepomechie, Nucl. Phys. B72 (1992) 597.

[40] H. J. de Vega and F. Woynarovich, Nucl. Phys. B 251 (1985) 439.

[41] C. J. Hamer, G. R. W. Quispel and M. T. Batchelor, J. Phys. A 20 (1987) 5677.

[42] J. L. Cardy J. Phys. A 17 (1984) L385.

[43] M. T. Grisaru, L. Mezincescu and R. I. Nepomechie, J. Phys. A 28 (1995) 1027.

[44] M. Jimbo, R. Kedem, T. Kojima, H. Konno and T. Miwa, "XXZ chain with a boundary", hep-th/9411112, to appear in Nucl. Phys. B. 